\title{
Pilots' willingness to operate in urban air mobility integrated airspace: a moderated mediation analysis
}

\author{
Lakshmi Vempati, Sabrina Woods, and Scott R. Winter
}

\begin{abstract}
Interest in advanced air mobility (AAM) and urban air mobility (UAM) operations for on-demand passenger and cargo transport continues to grow. There is ongoing research on market demand and forecast, community acceptance, privacy, and security. There is also ongoing research by National Aeronautics and Space Administration , Federal Aviation Administration, academia, and industry on airspace integration, regulatory, process, and procedural challenges. Safe integration of UAM and AAM will also require different stakeholder perspectives such as air traffic controllers, manned aircraft pilots, remote pilots, UAM operators, and the community. This research aimed to assess the willingness of manned aircraft pilots to operate in UAM integrated airspace based on airspace complexity and UAM automation level. In addition, a moderated mediation analysis was conducted using trust and perceived risk as mediators and operator type as a moderating variable. The results indicated that automation level influenced pilots' willingness to operate an aircraft in integrated airspace. A moderating effect of operation type on automation level and willingness to pilot an aircraft was also observed: professional pilots were more amenable to UAM operations with a pilot on board compared with remotely piloted operations. Results from the study are expected to inform airspace integration challenges, processes, and procedures for UAM integrated operations.
\end{abstract}

Key words: UAS, UAM, AAM, willingness to pilot an aircraft, WTPA, trust, perceived risk, moderated mediation.

Résumé : L’attention que suscitent les opérations de mobilité aérienne avancée (MAA) et de mobilité aérienne urbaine (MAU) dans le domaine du transport sur demande de passagers et de marchandises continue de croître. Des recherches sont en cours sur la demande et les prévisions du marché, l'acceptation sociale, la protection de la vie privée et la sécurité. La NASA ("National Aeronautics and Space Administration »), la FAA ("Federal Aviation Administration »), le milieu universitaire et l'industrie poursuivent également des recherches au niveau de l'intégration de l'espace aérien, de la réglementation, des processus et des défis au niveau des procédures. L'intégration en toute sécurité de la MAU et de la MAA devra également prendre en compte les perspectives des différents intervenants, comme les contrôleurs de la circulation aérienne, les pilotes d'aéronefs avec équipage, les pilotes à distance, les exploitants de la MAU et la collectivité. Cette recherche visait à évaluer la volonté des pilotes d'aéronefs avec équipage d'utiliser l'espace aérien intégré de MAU en fonction de la complexité de l'espace aérien et du niveau d'automatisation de la MAU. De plus, une analyse de médiation modérée a été effectuée en utilisant la confiance

Received 10 May 2021. Accepted 9 November 2021.

L. Vempati. The MITRE Corporation, McLean, VA 22102-7539, United States.

S. Woods. Federal Aviation Administration, Washington, DC 20591, United States.

S.R. Winter. Embry-Riddle Aeronautical University, Daytona Beach, FL 32114-3900, United States.

Corresponding author: Lakshmi Vempati (e-mail: lvempati@mitre.org).

(C) 2022 Winter and the MITRE Corporation. This work is licensed under a Creative Commons Attribution 4.0 International License (CC BY 4.0), which permits unrestricted use, distribution, and reproduction in any medium, provided the original author(s) and source are credited. 
et le risque perçu comme médiateurs et le type d'opérateur comme variable modératrice. Les résultats ont indiqué que le niveau d'automatisation influait sur la volonté des pilotes d'exploiter un aéronef dans un espace aérien intégré. Un effet modérateur du type d'opération sur le niveau d'automatisation et la volonté de piloter un aéronef a également été observé : les pilotes professionnels étaient plus réceptifs aux opérations de MAU avec un pilote à bord comparativement aux opérations télécommandées. Les résultats de l'étude devraient apporter un éclairage sur les défis, les processus et les procédures d'intégration de l'espace aérien pour les opérations intégrées de MAU. [Traduit par la Rédaction]

Mots-clés : système d'aéronef sans pilote (UAS), mobilité aérienne urbaine (MAU), mobilité aérienne avancée (MAA), volonté de piloter un aéronef (VPA), confiance, risque perçu, médiation modérée.

\section{Introduction}

During the 1970s and 1980s, developing unmanned aircraft vehicle (UAV) technology was primarily for bolstering military capabilities. Unmanned vehicles provided surveillance, intelligence, and war-fighting capability while ensuring the human was safely removed from imminent threat (Gupta et al. 2013). However, from the turn of the century, the scope and scale of UAV development and operation have rapidly evolved. It has now expanded to encompass commercial use such as land real estate surveillance and cargo lift, public use such as fire suppression and law enforcement, recreational use for hobbyists and remote craft builders, and even more recently, autonomous passenger airlift. Unmanned aircraft systems (UAS) comprise the vehicle and its command-and-control components (Gupta et al. 2013). Their rather prolific introduction into an already congested National Airspace System (NAS) has led to a scramble for both industry and the Federal Aviation Administration (FAA) to ensure safe integration.

It is guaranteed that the skies will get more crowded, providing a unique challenge for operators of both unmanned and manned vehicles. A 2013 Research and Innovative Technology Administration study projected that by the year 2035 the military, public, and commercial UAS fleet size will surpass 250000,175000 of which will be in the commercial marketplace (RITA 2013). For comparison, this is more than the entire general aviation fleet size as of 2019 (FAA 2020a, 2020b). This projection also does not include recreational use UAVs, which currently sit at more than 1.3 million registered with the FAA. UAS integration challenges include establishing robust legislation and regulations for integrated airspaces, developing procedures for pilot training and certification, and establishing both safety and security mechanisms for operations (RITA 2013). To help establish a structure for the transition, the FAA Reauthorization Act (H.R. 302, 2018), signed in 2018, provided new guidance on how UAVs would be managed. Most notable, Sections 3.4.5, 3.4.6, 3.4.7, and 3.4.8 of the Act address rulemaking for design certification standards, public-use activities, special authorization for beyond line of sight, and commercial delivery operations, respectively (FAA Reauthorization Act H.R. 302, 2018). Although the Act addressed the need to establish risk assessment in airspace integration, the human element of integration was not addressed. Additional research is needed to determine how the demand will impact air traffic management resources and capabilities, and the role manned aerial vehicle pilots will play in the transition. Pilot attitudes and willingness to fly in airspaces integrated with UAS will dictate how successful mixed-use airspaces will be in the future. This study aims to collect data to determine pilot trust and willingness to operate in a fully integrated environment.

There is ongoing interest in UAM operations in urban areas by companies such as Joby, Wisk, Archer, and others envisioning passenger-carrying operations by 2025 . These 
operations will likely be conducted in already congested and dense airspace close to major airports and near metropolitan areas. Further autonomy levels and human roles with automation might differ for different operators and operational requirements and capabilities. The objective of this study was to determine factors that influence manned aircraft pilots' willingness to operate in UAM integrated airspace. In the following subsections, we present the literature on autonomy levels, UAM concept of operations, trust in automation, manned perspectives on unmanned aircraft, challenges of see and avoid, airspace complexity and willingness framework, and perceived risk assessment. This is followed by a description of the current study, research methods, presentation of the results followed by a discussion of the results, practical applications and limitations, and finally some conclusions.

\section{Autonomy levels for unmanned systems}

Automated unmanned systems run via a defined set of parameters dictating what tasks are to be performed and when. In contrast, autonomous systems make their own decisions based on an algorithm or predetermined set of heuristics. Autonomy levels for unmanned systems were initially established by the automotive industry and are currently broken into five levels (Huang et al. 2005): Level 0 meaning no autonomy; Level 1 where autonomous systems are present (like thrust or altitude control), but the pilot is in control; Level 2 when multiple autonomous systems run simultaneously, but the pilot is in control; Level 3, the craft operates autonomously under certain conditions, but a pilot must monitor its progress; Level 4, the craft is autonomous in most situations and the pilot can take over but generally does not have to; and Level 5 where the craft is fully autonomous. The idea that UAS are unmanned is a bit of a misnomer in that current aviation technology is situated somewhere between Level 3 and 4, where the human monitors while the vehicle makes some or most of the decisions. A better definition might be that the pilot is simply not on board. Modern UAS are designed to receive input either from a remote manipulation from the pilot who is positioned elsewhere or by a software interface via a flight management computer that has been preprogrammed. They are typically a mix of both automated and autonomous systems.

As the average consumer becomes more and more accustomed to automation, the more companies feel they can continue developing and investing in autonomous technology. The latest emergence is that of the urban air mobility (UAM) category. Companies such as EHang in China, Volocopter based in Germany, and Joby Aviation in the United States have made it their goal to revolutionize air mobility by providing efficient, electric, autonomous passenger flight all on demand. Each advertises a desire to eliminate the possibility of the introduction of hazards due to human error. The goal is to go Level 5-the vehicle is in complete control and has full decision-making authority. Currently, however, UAM operations are evolving from having a pilot on board to a remote pilot. UAM automation falls into one of three categories: human-within-the-loop (HWTL), meaning the human is always in direct control of the automation; human-on-the-loop (HOTL), meaning the human is supervising the automation and can take control when desired; or human-over-the-loop (HOVTL), meaning the human is passively monitoring the automation and can step in if cued to do so (Federal Aviation Administration (FAA) Next Generation Office (NextGen) 2020). For this research, the two UAM automation levels, one with pilot on board (HWTL and (or) HOTL), and remote pilot monitoring (HOVTL) are considered.

\section{UAM concept of operations (CONOPS)}

In collaboration with the National Aeronautics and Space Administration (NASA) and its industry partners, the FAA has published version one of its concept of operations (CONOPS) 
for UAM. The guide intends to lay the foundation for how unmanned systems involving commercial cargo and passenger lift will integrate and operate in the NAS. Because of the relatively low altitude and small profile of these types of UAS, it is envisioned that UAM will operate within the same parameters as helicopters, using their existing routes and infrastructure (Federal Aviation Administration (FAA) 2020). Many in the industry view the CONOPS document as merely a starting point and have expressed interest in further developing the guidance. Questions regarding zoning, sequencing, letters of agreement, engagement levels of local law enforcement, and the disposition of noncompliant entities remain. What the CONOPS does address, however, is the need for additional "analysis, simulation, demonstration, and community engagement" (Federal Aviation Administration (FAA) 2020 p. 39), elements that further research of attitudinal and perception data will surely help to inform.

\section{Trust in automation}

Trust is defined as the extent to which one entity believes in the reliability, truth, ability, and (or) strength of someone or something. Trust is underpinned by the state of willingness and relative security the entity has regarding the subject (Jøsang and Presti 2004). Evaluating trust in autonomous systems has been the focus of several different studies covering a large swath of domains. As technology advances, its success is mainly contingent on establishing and maintaining mutual trust (Beer et al. 2014). A pilot's trust in another pilot stems from the idea that there is a shared high level of mental and physical standards achieved to operate an aircraft (Cantwell 2009). A pilot's trust in automation is directly tied to whether they believe the automation's capability level exceeds their own (Lee and Moray $1992,1994)$. For a pilot to view and value an autonomous system as a separate, consummate peer worthy of trust, all the expectations for both other pilot and automation must be met.

For the human-automation system to work effectively, there must be a balance between the efficacy of the automation and the engagement level of the human. Automation with a questionable performance record, or that is known to produce false alarms, significantly lowers trust in the user (Muir 1994). Mistrust in automation often leads to misuse or even disuse, leading to a mishap (Parasuraman and Riley 1997; Dixon et al. 2007). This result would be regardless of the automation serving as a tool or in the role of peer.

Conversely, a pilot heavily dependent on automation runs the risk of becoming complacent due to underload. Too much trust in automation and autonomous systems could lead to lowered vigilance and supervision (Parasuraman et al. 1993, Moray and Inagaki 1999). This overreliance can take the human out of the information loop, decrease automation awareness, and introduce bias (Endsley 1996). Automation bias can have the unintended consequence of instilling far more mistrust in the system should it fail than were the pilot to have made the same error. Automation is simply not allowed the same latitude a human is in these circumstances.

\section{Manned perspectives on unmanned operations}

Many unique human factors considerations help to form an individual's positive or negative attitude towards UAS operations. For instance, a positive attitude might develop from the idea that automated devices are perceived as being far more capable, indefatigable, and vigilant than any human (Lee and Moray 1992, 1994). A negative attitude might come from a pilot's mistrust in their ability to see and avoid the unmanned vehicle, that the vehicle will likewise steer clear of the manned operation, or in the perceived skill level of the operator manipulating the unmanned vehicle.

Several studies have suggested that familiarity and failsafe play prominent roles in developing positive attitudes. Butakov and Ioannou (2015) postulated that if the behavior 
and design of an automated system are like that of a regular system, users are more likely to trust it. Butakov and Ioannou (2015) suggested that if an unmanned vehicle behaves and operates in the same manner expected and familiar to a manned aircraft, the latter's pilots might hold a more favorable opinion. Richards and Edgell (2017) surveyed 196 participants consisting of air traffic controllers, aircraft pilots, and UAS pilots. Richards and Edgell (2017) determined that a large percent felt full automation (Level 5) would be acceptable if there were sufficient redundancies $(14.8 \%)$ or a human $(13.8 \%)$ in place ready to take back control should an issue arise. Richards and Edgell (2017) also identified that aircraft pilots expressed a negative attitude toward small UAS integration, although large UAS were perceived slightly more positively. However, it must be mentioned that this study was conducted in the wake of a mid-air collision between an alleged small UAS and a British Airways flight on approach to Heathrow, which might have influenced the outcome. The collision was later determined not to be with an unmanned vehicle. In the same study, pilots of unmanned vehicles expressed frustration that they are not seen as being commensurate with their manned vehicle counterparts. This lends some credence to the idea that mistrust can develop on behalf of the manned vehicle pilot regarding the skill level and experience of the UAS pilot.

\section{The challenge of see and avoid}

The challenges of operating in an airspace also being used by autonomous vehicles lie in the perceived ability to detect and avoid one another. A pilot's trust in operating safely in the NAS is mainly contingent upon this idea that all users can and will see and avoid. In instrument meteorological conditions, air traffic is controlled by automated and human systems that relay information between air traffic control and aircraft. This system is designed to provide alerts if there are potential conflicts; however, only large, Part 47, N-registered UAS are currently integrated (Aircraft Registration 2020).

See and avoid becomes more imperative in visual flight rules flight, and its concept is detailed in the FAA's Advisory Circular (AC) 90-48D (FAA 2016). The AC was written to alert pilots to the potential for mid-air collisions and emphasize the need to recognize other aircraft and determine their orientation to maintain visual separation (FAA 2016). The AC highlights vigilance, planning, scanning, and responsibility and instructs how one should scan and what resources are available to aid in the process. While the AC is a valuable document, it is limited because it was explicitly written for manned aircraft pilots. The assumption here is that pilots could use this tool to understand and trust that all other manned aircraft pilots would engage in similar behaviors. The likelihood that separation would be maintained increases. The AC addresses UAS only because manned aircraft pilots should avoid them, and it does not address how the UAS very presence might diminish the concept. Although a manned aircraft pilot has access and can use all five senses, a remote pilot operating a UAS is limited to just visual cues, which could present an additional barrier to see and avoid (Pestana 2011). Further, in Richards and Edgell's (2017) study, a large percentage of surveyed pilots felt that they had the right of way in visual meteorological conditions when confronted with UAS. These results are interesting and bolster the idea that manned aircraft are considered more vulnerable and therefore should have the rightof-way, but it is a bit at odds with the general skepticism over the ability of UAS to see and avoid. It remains to be seen how these attitudes might shift when confronted with UAM passenger transport systems with known occupants on board.

\section{Airspace complexity}

The basic structure of airspace is broken into regulated and nonregulated, with four types in each category: controlled, uncontrolled, special use, and other. The nature of 
operations in the airspace-its traffic volume, type of traffic, and the level of effort it takes to monitor and manage traffic - is what defines its complexity. The predicted growth of air traffic throughout the NAS will change airspace complexity and impose a significantly increased demand on air traffic management systems. Several studies have investigated this impact to understand its effect on air traffic controller workload and pilot aeronautical decision-making.

Although the complexity of airspace has a proven positive correlation to workload for both the air traffic controller and the pilot, complexity should not be synonymous with workload. As workload increases, however, the ability to accurately predict and manage complexity is challenged, leaving the airspace vulnerable to human error (Radišić et al. 2020). Kopardekar et al. (2008) sought to develop quantifiable metrics in determining complexity. The thought here is that if a value can be assigned, then control measures can be developed to mitigate the risk to an acceptable level. Future mitigations might include staffing assessments and reassignments, reconfiguration of airspaces, and the development of new deconfliction tools. It is inevitable that like UAS integration, current controller and pilot perceptions will dictate how well these changes will be received and how successful they will eventually be. The establishment of these mitigations will play a vital role in the ability to instill trust in the continued safety of the NAS.

\section{Willingness framework and perceived risk assessment}

To understand a pilot's level of willingness to operate in mixed-use airspaces, one must understand the risks and trust factors associated with the decision. Willingness is defined as the state of being prepared to do something without reluctance. One's willingness plays a crucial role in their reasoned actions because it reflects how likely they are to perform a specific behavior (Ajzen 1991). Ajzen and Fishbein (1980) postulated that there is a high correlation between expressed attitude and actual behavior. For scenario-based research, this means that how a person reports they feel about a topic is likely to correspond to how they would behave if the circumstances were to come to fruition.

A thorough risk assessment is warranted to build the effective mitigations needed to manage complexity and traffic density. Simply stated, a hazard is any condition existing within the environment that has the potential to cause injury or damage. Risk is determined by one's likelihood of encountering the hazard and the potential severity of the outcome from that encounter. Given these descriptions, UAS operations pose a hazard just as any other manned operation would when operating in the NAS. However, the perception changes are in determining the risk that is inherent to UAS versus that of their manned counterparts.

Jøsang and Presti (2004) identified risk and trust as the two facets that facilitate a person's ability to decide and then act upon it. While Jøsang and Presti's (2004) focus was on economic and transactional trust and risks, their findings have application here. The manned aircraft pilot's willingness to operate in the same airspaces as UAS is entirely dependent on their ability to assess the risk it poses, combined with the level of trust that they have in the mitigations that are currently established to manage the risk. As discussed in the sections, "Trust in automation" and "See and avoid", the concern of operating within mixed-use airspaces is the likelihood that a manned aircraft will come into contact with UAS, and one or both vehicles will fail to see and avoid.

\section{Current study}

Vempati et al. (2021) studied pilot perceptions and willingness to pilot an aircraft in UAS integrated airspace and found that airspace classification was a major factor that influenced pilot's willingness to operate. Further, there were differences based on the different 
Fig. 1. Conceptual research framework. $\mathrm{H}_{\mathrm{A1}}$ : There will be a significant difference in pilots' willingness to pilot an aircraft based on airspace complexity. $\mathrm{H}_{\mathrm{A} 2}$. There will be a significant difference in pilots' willingness to pilot an aircraft based on the urban air mobility (UAM) automation level. $\mathrm{H}_{\mathrm{A} 3}$ : There will be significant interaction between airspace complexity and UAM automation level. $\mathrm{H}_{\mathrm{A} 4}$ : There will be a significantly moderated mediation between airspace complexity and willingness to pilot. $\mathrm{H}_{\mathrm{A} 5}$ : There will be a significantly moderated mediation between UAM automation level and willingness to pilot.

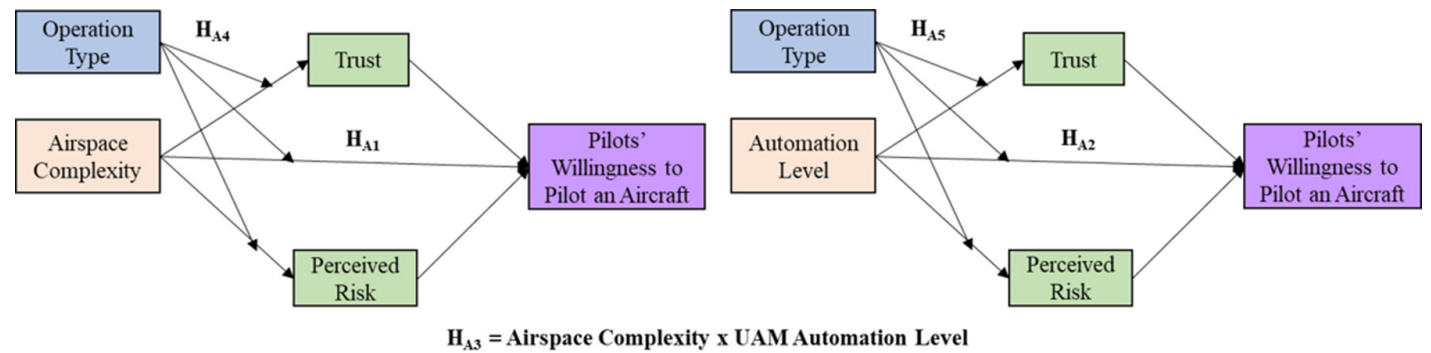

classes of airspace and the type of operations being conducted. The current study expands on Vempati et al. (2021) to further assess manned aircraft pilots' willingness to operate in UAM-integrated airspace based on airspace complexity (low, medium, or high) and UAM automation level (pilot onboard monitoring or remote pilot monitoring). Also, we assess the moderated mediation between airspace complexity/UAM Automation level and willingness to pilot based on the type of manned operations (recreational or commercial purpose), trust in automation, and perceived risk. The conceptual model is depicted in Fig. 1, and we proposed the following hypotheses.

$\mathrm{H}_{\mathrm{A} 1}$ : There will be a significant difference in pilots' willingness to pilot an aircraft based on airspace complexity.

$\mathrm{H}_{\mathrm{A} 2}$ : There will be a significant difference in pilots' willingness to pilot an aircraft based on the UAM Automation level.

$\mathrm{H}_{\mathrm{A} 3}$ : There will be significant interaction between airspace complexity and UAM Automation level.

$\mathrm{H}_{\mathrm{A} 4}$ : There will be a significantly moderated mediation between airspace complexity and willingness to pilot.

$\mathrm{H}_{\mathrm{A} 5}$ : There will be a significantly moderated mediation between UAM Automation level and willingness to pilot.

\section{Methods}

\section{Participants}

Four hundred and one responses were collected from the U.S. pilot population using an electronic questionnaire hosted on Google Forms. Embry Riddle Aeronautical University guidelines were adhered to, prior to conducting this electronic questionnaire, by filing an application seeking the approval of Internal Review Board (IRB). Four different pilot organizations or newsletter sources were used to collect the data: Curt Lewis Flight Safety Newsletter, FAA Safety Briefing Magazine, General Aviation News, and Smokehouse Pilots Club. Participants were also encouraged to share the survey with other interested pilots or pilot groups.

Of the 401 responses, two participants did not consent or did not qualify, and a further 19 participants did not complete all or some of the scale-related questions. The incomplete response rate was less than 5\%, and the responses from 380 participants (330 males, 34 females, 16 who did not provide gender information) completed all questions used in the 
Table 1. Pilot participant characteristics by geographical region in the United States.

\begin{tabular}{ll}
\hline Geographical region & \% of participants \\
\hline Southern region & $22 \%$ \\
Midwest region & $19 \%$ \\
Pacific region & $16 \%$ \\
Mid-Atlantic region & $15 \%$ \\
Southwest region & $11 \%$ \\
Rocky Mountain region & $8 \%$ \\
New England region & $7 \%$ \\
Other & $2 \%$ \\
\hline
\end{tabular}

final analysis. Almost $86 \%$ of the participants were White or Caucasian and evenly distributed across the country's major regions. A summary of the participants by geographical region is provided in Table 1.

Materials, stimuli, and procedure

Responses from the pilot population were collected using a factorial survey experiment. Participants were provided a list of questions and sample scenarios. They were randomly assigned different UAM automation levels (IV1, between factor) and airspace complexity level (IV2, between factor), to assess their Trust (Mediator 1), Perceived Risk (Mediator 2), and Willingness to Pilot an Aircraft (DV) in UAM integrated airspace. The type of operation was used as a moderating variable. Demographics and other information such as age, gender, and experience level were collected. In addition, pilots' opinions on operating under the specified scenario were also collected.

\section{Measurement scales}

The willingness to pilot an aircraft (WTPA) scale developed by Rice et al. (2020) was used in this study for the dependent variable. It has been shown to be reliable and valid. The study also used two scales to determine the mediating role of trust and perceived Risk on WTPA. The trust in automated systems developed by Jian et al. (2000) and the risk perception scale developed by Clothier et al. (2015) were used in the moderating mediation analysis. The adapted scales used in the current study are provided in Appendix A.

\section{Design and data analysis}

This research study used a quantitative methodology and an experimental design with a moderated mediation analysis. IBM SPSS Statistics 26 (2019, Armonk, NY: IBM Corp) was used to complete the data analysis and the Hayes PROCESS plugin to conduct the moderated mediation assessment (Hayes 2018).

\section{Results}

\section{Descriptive statistics and initial data analysis}

The study used a sample of 380 participants, of whom there were 34 female pilots with a mean age of $51.6(\mathrm{SD}=14.4), 330$ male pilots with a mean age of $56.2(\mathrm{SD}=14.8)$, and 16 who did not provide gender information. The mean age of all pilots was $55.6(\mathrm{SD}=14.8)$. Fifty-seven percent of the pilots flew recreationally with average flight hours 2849 (SD = 5 630), while the remaining 44.4 percent flew for business or commercial purposes with average flight hours 8768 ( $S D=8$ 027). Each participant was randomly assigned one of the six hypothetical scenarios-each containing one level of airspace complexity (high, medium, or low) and one level of automation level (pilot on board or remote pilot monitoring). Table 2 provides some pilot demographics for the six hypothetical scenarios. 
Table 2. Pilot demographics for the six conditions.

\begin{tabular}{|c|c|c|c|c|c|c|c|c|}
\hline \multirow[b]{2}{*}{ Airspace Complexity } & \multicolumn{4}{|c|}{ Pilot on board the urban air mobility aircraft } & \multicolumn{4}{|c|}{$\begin{array}{l}\text { Remote pilot monitoring the urban air mobility } \\
\text { aircraft }\end{array}$} \\
\hline & $\begin{array}{l}\text { No. of } \\
\text { participants }\end{array}$ & $\begin{array}{l}\text { Mean } \\
\text { age, } y\end{array}$ & $\begin{array}{l}\text { Mean } \\
\text { flight } \\
\text { hours }\end{array}$ & $\begin{array}{l}\text { Participants, } \\
\text { recreational \% } \\
\text { (professional \%) }\end{array}$ & $\begin{array}{l}\text { No. of } \\
\text { participants }\end{array}$ & $\begin{array}{l}\text { Mean } \\
\text { age, } y\end{array}$ & $\begin{array}{l}\text { Mean } \\
\text { flight } \\
\text { hours }\end{array}$ & $\begin{array}{l}\text { Participants, } \\
\text { recreational \% } \\
\text { (professional \%) }\end{array}$ \\
\hline High (Class B \& Class C) & 49 & 54 & 6955 & $51(49)$ & 101 & 56 & 5568 & $54(46)$ \\
\hline Medium (Class D \& Class E) & 52 & 57 & 5147 & $58(42)$ & 69 & 55 & 4626 & $68(32)$ \\
\hline Low (Class G) & 53 & 59 & 5907 & $58(42)$ & 56 & 53 & 4,195 & $54(46)$ \\
\hline
\end{tabular}


Table 3. Descriptive statistics for the willingness to pilot an aircraft scale for the six conditions.

\begin{tabular}{|c|c|c|c|c|c|c|}
\hline \multirow[b]{2}{*}{ Airspace complexity } & \multicolumn{3}{|c|}{$\begin{array}{l}\text { Pilot on board the urban } \\
\text { air mobility aircraft }\end{array}$} & \multicolumn{3}{|c|}{$\begin{array}{l}\text { Remote pilot monitoring } \\
\text { the urban air mobility } \\
\text { aircraft }\end{array}$} \\
\hline & Mean & SD & Median & Mean & SD & Median \\
\hline High (Class B \& Class C) & 0.399 & 1.289 & 0.860 & -0.124 & 1.065 & 1.000 \\
\hline Medium (Class D \& Class E) & 0.223 & 1.200 & 0.430 & 0.089 & 1.111 & 0.570 \\
\hline Low (Class G) & 0.437 & 1.131 & 0.710 & 0.005 & 1.112 & 1.000 \\
\hline
\end{tabular}

Fig. 2. Pairwise comparison of automation level by airspace complexity. Mean differences of estimated marginal means with standard error bars are depicted for the average willingness to pilot an aircraft score.

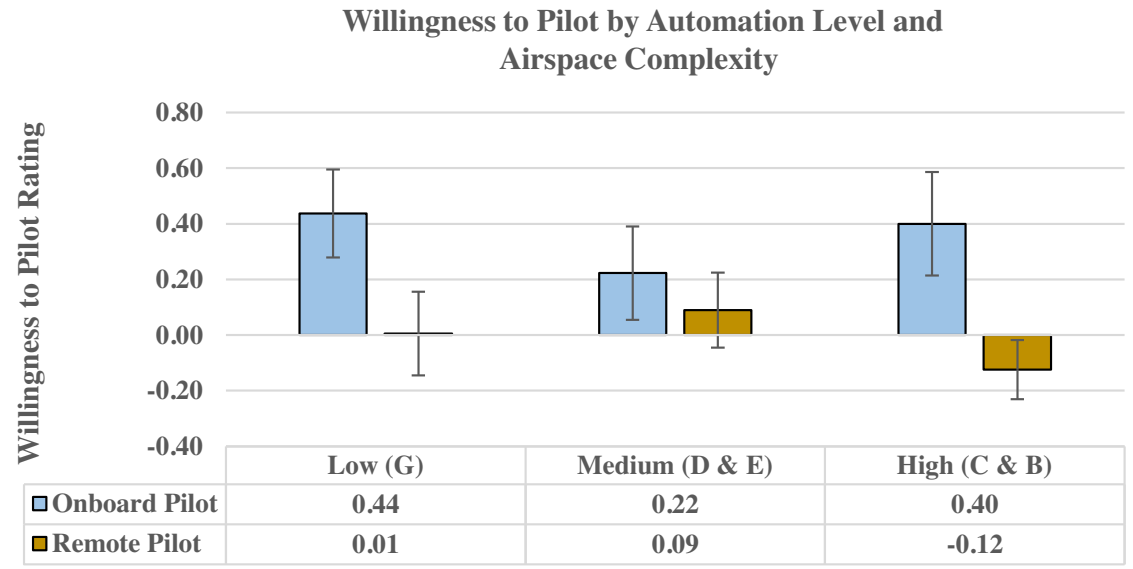

The three data-related assumptions of no significant outliers, homogeneity of variances, and normality required for two-way analysis of variance (ANOVA) analysis were also verified before conducting the analysis. Descriptive statistics for the WTPA scale for the six hypothetical scenarios are shown in Table 3.

\section{Inferential statistics}

For the main data analysis, we conducted a $2 \times 3$ between-participants factorial ANOVA with: UAM automation level, a categorical variable with two levels (pilot onboard monitoring or remote pilot monitoring); airspace vcomplexity, a categorical variable with 3 levels (high (B \& C), medium (D \& E) \& low (G)); and one dependent variable, WTPA, a continuous, 7-point Likert scale. The results identified a statistically significant main effect for automation level, $F(1,373)=9.045, p=0.003, \eta_{\mathrm{p}}{ }^{2}=0.024$. There was no significant main effect for airspace complexity, $F(2,373)=0.171, p=0.843, \eta_{\mathrm{p}}{ }^{2}=0.001$, nor a significant interaction between automation level and airspace complexity $F(2,373)=0.980, p=0.376$, $\eta_{\mathrm{p}}{ }^{2}=0.005$.

The simple main effects for levels of automation level by airspace complexity indicated differences between pilot on board and the remote pilot in the low complexity and high complexity airspace conditions, where participants were significantly more willing to pilot with a human pilot than a remote pilot in those airspace classifications. These findings are depicted in Fig. 2. 
Fig. 3. Moderation effect of operation type on automation level and willingness to pilot an aircraft (WTPA).

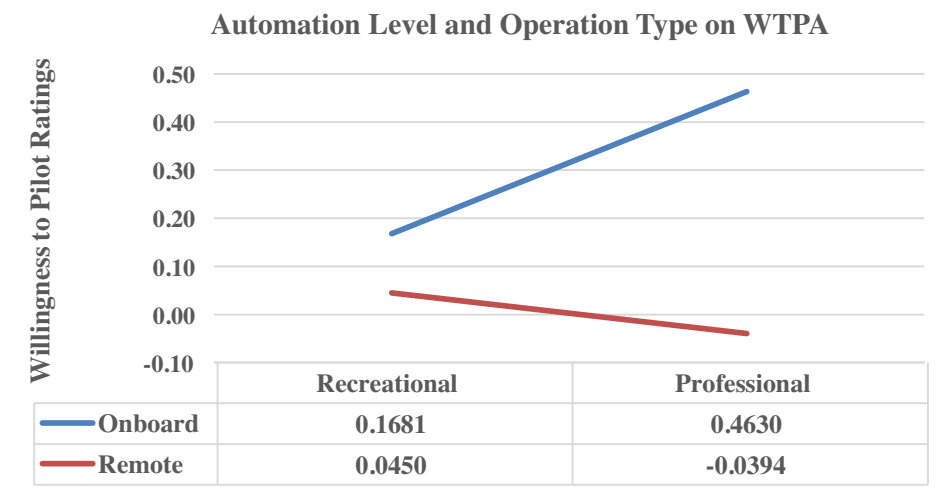

Next, the PROCESS module developed by Hayes (2018) and available as a plugin within SPSS was used to conduct the moderated mediation analysis. The moderated mediation was conducted using 10000 bootstrapped samples IV1 or IV2. The results of this analysis indicated no significant moderated mediation of operation type, trust or risk on automation level, or airspace complexity

No significant moderating mediation effect of airspace complexity was observed on WTPA. No main effects of airspace complexity or interaction effects with trust and risk were observed. Trust and risk did have a direct effect on WTPA. A significant moderation was found between automation level and the type of operation $(p=0.0213)$ on WTPA, as shown in Figure 3.

Differences were observed between recreational and professional pilots for situations when a pilot was on board the UAM, where professional pilots were significantly more willing to pilot when a human was on board than recreational pilots. No other relationships were significantly different. Table 4 depicts the simple main effects of this moderation. A pairwise comparison of automation level and operation type indicated significant differences between commercial or business use pilots and recreational pilots for automation level with an onboard pilot, as shown in Table 4.

\section{Qualitative responses}

Pilot perspectives were also collected using open-ended questions to provide more detailed responses and narratives on their concerns, expectations, and other information about operating in UAM integrated airspace. Many of the concerns centered around human factors issues about the pilot on board and complacency, automation issues related to the handling of off-nominal situations, congestion, and limits to airspace with increasing traffic density, communication, navigation and surveillance issues such as see and avoid, communication with air traffic control, and other traditional airspace users. A few example narratives are provided below:

"Good visibility conditions at controlled airports would be the lowest risk level. The technology still needs to prove itself in congested, downtown, urban areas but my greatest concern is congestion. If the end game is to replace, even one-in-four, Uber ground vehicles, then city centers will look like beehives. Reduce the visibility and throw in an inflight malfunction and the "beehive" is in crisis as the prototypes I've heard of don't have tremendous loiter time or range." 
Table 4. Pairwise comparison of automation level and operation type.

95\% Confidence interval for difference $^{b}$

\begin{tabular}{|c|c|c|c|c|c|c|c|}
\hline \multirow[b]{2}{*}{ Automation level } & \multirow[b]{2}{*}{ Operation type, I } & \multirow[b]{2}{*}{ Operation type, II } & \multirow{2}{*}{$\begin{array}{l}\text { Mean } \\
\text { Difference (I-II) }\end{array}$} & \multirow{2}{*}{$\begin{array}{l}\text { Standard } \\
\text { error }\end{array}$} & \multirow[b]{2}{*}{ Significance $^{b}$} & \multicolumn{2}{|l|}{ Сmeience } \\
\hline & & & & & & Lower bound & Upper bound \\
\hline Onboard pilot & Commercial/business use & Recreational purpose only & $.440^{*}$ & 0.184 & $0.017^{*}$ & 0.077 & 0.802 \\
\hline Remote pilot & Commercial/business use & Recreational purpose only & -0.091 & 0.153 & 0.550 & -0.391 & 0.209 \\
\hline
\end{tabular}

Note: Based on estimated marginal means.

${ }^{*}$ The mean difference is significant at 0.05 .
${ }^{b}$ Bonferroni adjustment for multiple comparisons. 
"I believe that engineers can design reliable, efficient, reasonably safe systems. My main concerns with integration of UAM and UAS into the NAS have to do with philosophical implications. If the skies above Class $C$ and $B$ airports become filled with drones, the ability of small commercial operators and private individuals to access that airspace could become further limited. Demand for the air mobility may displace other users of the airspace, or subject current users to even more stringent control (not really applicable within Class B, but in the area underneath and in the vicinity of Class B). The see-and-avoid systems and reversionary/redundant navigation in case of system failure in UAM will have to be absolutely flawless for me to have no reservations for safely operating near the systems."

"I am greatly concerned that the average person today seems to feel that autonomous systems are superior because of the lack of human participation, and that manned systems are inferior because humans will always err. The fact of the matter is that an autonomous system is only as perfect as those who have designed it, which means that it is no more perfect than a manned system. My fear, though, is that because of flawed public perception that autonomous systems are automatically safer than manned ones, public pressure will influence regulators and lawmakers to give increasing priority to UAMs, eventually forcing me and my manned aircraft from the skies."

"Presuming the UAM operations are certified under the same rules and scrutiny as crewed aircraft and the UAM components have appropriate levels of redundancy and safety integrity then the issue is going to be traffic density and collision avoidance in high density Class B/C airspace."

\section{Discussion}

While in their early implementation, UAS were primarily segregated from manned operations (Gupta et al. 2013). By 2025, projections anticipate considerable growth in the fleet size of public use and commercial UAS and UAM operations (RITA 2013). The current study builds upon prior research related to the integration of UAS into the NAS (Vempati et al. 2021). The purpose of the current study was to assess the willingness to pilot when UAM vehicles were integrated into the airspace with crewed flight operations. These effects were examined based on the level of airspace complexity and automation level of the UAM vehicle. In addition, the moderating role of the operation type and possible mediating effects of trust and perceived risk were examined for a possible moderated mediation of any existing relationships.

Hypothesis $\mathrm{H}_{\mathrm{A} 1}$ predicted a significant difference in willingness to pilot based on airspace complexity. The data from the current study did not support this hypothesis. While a prior study related to the use of unmanned aircraft systems found some difference based on airspace classification (Vempati et al. 2021), in the current study, the airspace classifications were grouped by their level of complexity (low, medium, and high); however, the given scenario and pilot-anticipated risk factors might have had some influence on their willingness. Given the differences between Vempati et al. (2021) and the current study, further research should investigate any differences in the role of airspace in the pilot's willingness to operate when UAS or UAM vehicles are present in the surrounding airspace. One possible explanation for the differences in the two studies is the alteration between the use of individual airspace classifications (Vempati et al. 2021) or the use of airspace groupings, as was conducted in the current study. It is also possible that because of the novelty of integrating UAS and UAM into the airspace with manned operations, the airspace classification has little impact on the pilot's willingness to pilot.

$\mathrm{H}_{\mathrm{A} 2}$ predicted that willingness to pilot would be affected by the automation level of the UAM vehicle. The findings supported this hypothesis. In general, pilots were more willing 
to pilot when a human pilot was on board the vehicle compared with when the vehicle was remotely piloted. As UAM vehicles are developed, their implementation with manned operations will require careful coordination. From a willingness perspective, other pilots may be more accepting of UAM deployment if humans are on board, especially as the first level of implementation begins (Beer et al. 2014). The highly automated level of operation of these vehicles will provide a unique aspect to what many manned pilots are familiar with. Thus, using an onboard pilot may assist in the overall integration and acceptance of these vehicles into the national airspace system (Cantwell 2009).

$\mathrm{H}_{\mathrm{A} 3}$ predicted a significant interaction between the airspace complexity and automation level. The data from the current study did not support this prediction, and this finding is somewhat different than an earlier study related to UAS integration (Vempati et al. 2021). Vempati et al. (2021) found a significant interaction between the operation type and airspace classification; however, the operation types were either remotely piloted or autonomous, differing from the human on board or remotely piloted scenarios in the current study. As with $\mathrm{H}_{\mathrm{A} 1}$, the groupings of airspace complexity may not have provided for the level of fidelity necessary to distinguish between differences in airspace complexities. Further research should seek to determine if any differences exist based on the airspace classification.

Hypotheses $\mathrm{H}_{\mathrm{A} 4}$ and $\mathrm{H}_{\mathrm{A} 5}$ both predicted moderated mediation would be found between the two independent variables and dependent variable. $\mathrm{H}_{\mathrm{A} 4}$ was not supported by the results of the current study. $\mathrm{H}_{\mathrm{A} 5}$ was partially supported. While a moderated mediation was not found, a significant moderation was shown to exist between automation level and operation type on willingness to pilot. The data indicate that professional pilots are significantly more willing to pilot than recreational pilots when a human pilot is on board the UAM than when it is remotely operated. This finding could be explained by several aspects unique to professional pilot operations. First, professional pilots tend to primarily fly into larger airports, with denser traffic flows, in high-speed and high-performance aircraft. The uncertainty that could be caused by integrating UAM operations into this environment may provide one possible explanation of this finding. Also, professional pilots may be considering the number of passengers they have on board, typically many more than may be on board a recreational pilot's aircraft. The safety of their passengers may be another aspect that influences this significant moderation. This further suggests the need for inclusion of different risk factors that might influence pilot's intentions and attitudes towards operating in mixed-use airspace.

\section{Practical applications}

There are several practical applications of the results of the current study. First, there appears to be a greater willingness to pilot when a human pilot is on board the UAM compared with remote operations. As UAM vehicle manufacturers are considering the development of their vehicles and their initial deployment, incorporating a human operator may likely increase the willingness of pilots to accept or operate around these vehicles. As confidence is gained in UAM usage from the manned pilot population, remote operation or autonomous operations may be more feasible. In addition, manufacturers should promote their products, specifically related to safety aspects, and familiarize pilots with how these new vehicles can be incorporated into the national airspace while integrated with traditional operations.

Professional pilots also indicated a stronger willingness when human pilots were on board the UAM. Consideration should be given to when and where these UAM vehicles will be integrated into the airspace. There may be some advantages of completing the first operations in less dense airspace without congestion in areas where large commercial aircraft 
typically operate. Participation in events, such as the national campaign being led by NASA, may be another avenue to demonstrate the usability, feasibility, and safety surrounding the deployment and incorporation of UAM into the national airspace system. These demonstrations can provide valuable information to traditional pilots to familiarize them with these newly developed UAM vehicles.

\section{Limitations}

There were several limitations to the current study. First, a convenience sample was used to collect participants, and it was limited to those who received and participated in reviewing electronic newsletters and promotions. This sample restricts the generalizability of the findings, and future research should attempt to gain access to a broader selection of pilots. The classification of the airspace categories may have failed to provide enough fidelity to note distinctions among these airspace classifications, especially since the current study's findings differ from a preceding study. The nature of perceived risk such as risk of failing, risk of automation failing, or risk of remote pilot failure can further influence pilots' attitudes for operating in different classifications of airspace. Further research should investigate the role airspace classification plays in willingness to pilot as it relates to UAS and UAM including consideration of different risk factors that might influence intent. Lastly, while perceived risk and trust were not identified as significant mediators in the current study, future research should investigate their relationships with the willingness to pilot in the context of UAM. Although not significant as mediators, they may be part of a broader statistical model that helps to understand factors related to a pilot's willingness to operate with integrated UAS and UAM operations.

\section{Conclusions}

The focus of the current research study was to understand factors that would influence manned aircraft pilots' willingness to operate in UAM integrated airspace. A $2 \times 3$ between-factors ANOVA using airspace complexity (low, medium, and high), and automation level (pilot on board or remotely piloted) was used to assess the willingness to pilot an aircraft scale developed by Rice et al. (2020). In addition, two mediating variables, trust and perceived risk, and one moderating variable, operation type (recreational or commercial purpose), were used to understand the moderating mediation effects on those relationships. The results of the analysis found that while airspace complexity had no impact and no interaction effects were observed, automation level clearly influenced pilots' WTPA. Although no mediating effects were observed, there was clearly a moderating effect of operation type on automation level and WTPA. Professional pilots were more amenable to UAM operations with a pilot on board compared with remotely piloted operations. Further research is necessary to better understand the impacts of airspace complexity on pilots' willingness to operate in UAS and UAM integrated airspace and the role trust and perceived risk might play in influencing pilots' perceptions and willingness to operate UAS and UAM integrated airspace. Thus, incorporating additional factors such as UAM traffic density, proximity of manned and unmanned operations, other airspace related features, risk appetite, and autonomy levels might provide further understanding of pilots' intentions for operating in mixed-use airspace.

\section{References}

Aircraft Registration Unmanned Aircraft. 2020. Federal Aviation Administration. Available from https:// www.faa.gov/licenses_certificates/aircraft_certification/aircraft_registry/ua/.

Ajzen, I. 1991. The theory of planned behavior. Org. Behav. Hum. Dec. Proc. 50(2): 179-211. doi: 10.1016/0749-5978(91) 90020-T. 
Ajzen, I., and Fishbein, M. 1980. Understanding attitudes and predicting social behavior. Engle-wood-Cliffs, Prentice-Hall, N.J.

Beer, J., Fisk, A.D., and Rogers, W.A. 2014. Toward a framework for levels of robot autonomy in human-robot interaction. J. Hum.-Robot Interact. 3(2): 74. doi: 10.5898/JHRI.3.2.Beer. PMID: 29082107.

Butakov, V., and Ioannou, P. 2015. Driving autopilot with personalization feature for improved safety and comfort. 18th International Conference on Intelligent Transportation Systems, pp. 387-393, IEEE.

Cantwell, H.R. 2009. Operators of air force unmanned aircraft systems: Breaking paradigms. Air Space Power J. 23 (2): 67.

Clothier, R.A., Greer, D.A., Greer, D.G., and Mehta, A.M. 2015. Risk perception and the public acceptance of drones. Risk Anal., 35(6): 1167-1183. doi: 10.1111/risa.12330. PMID: 25689883.

Dixon, S., Wickens, C.D., and McCarley, J.M. 2007. On the independence of reliance and compliance: Are false alarms worse than misses? Hum. Factors, 49: 564-572. doi: 10.1518/001872007X215656. PMID: 17702209.

Endsley, M.R. 1996. Automation and situation awareness. In Human factors in transportation. Automation and human performance: Theory and applications. Edited by R. Parasuraman and M. Mouloua. Lawrence Erlbaum Associates, Inc. p. 163-181.

Federal Aviation Administration [FAA]. 2016. Pilots' role in collision avoidance. Advisory Circular AC 90-48D. Department of Transportation. Available from https://www.faa.gov/documentLibrary/media/Advisory_Circular/ AC_90-48D.pdf.

FAA Reauthorization Act of 2018, H.R. 302. 2018. Available from https://www.congress.gov/bill/115th-congress/housebill/302/text.

Federal Aviation Administration [FAA]. 2020. General Aviation and Part 135 Activity Surveys - CY 2019. (2020) Available from https://www.faa.gov/data_research/aviation_data_statistics/general_aviation/CY2019/.

Federal Aviation Administration [FAA] Next Generation Office [NextGen]. 2020. Concept of operations, v1.0: Urban air mobility. Department of Transportation. Available from https://nari.arc.nasa.gov/sites/default/files/ attachments/UAM_ConOps_v1.0.pdf.

Gupta, S.G., Ghonge, D., and Jawandhiya, P.M. 2013. Review of unmanned aircraft system (UAS). Inter. J. Adv. Res. Comp. Eng. Technol. (IJARCET), 2.

Hayes, A.F. 2018. Introduction to mediation, moderation, and conditional process analysis second edition. The Guilford Press, New York, NY.

Huang, H.M., Pavek, K., Novak, B., Albus, J., and Messin, E. 2005. A framework for autonomy levels for unmanned systems (ALFUS). Proc. AUVSI's Unman. Sys. North Am. 849-863.

Jian, J.Y., Bisantz, A.M., and Drury, C.G. 2000. Foundations for an empirically determined scale of trust in automated systems. Inter. J. Cogn. Ergon. 4(1): 53-71. doi: 10.1207/S15327566IJCE0401_04.

Jøsang, A., and Presti, S.L. 2004. Analysing the relationship between risk and trust. In International conference on trust management. Springer, Berlin, Heidelberg. pp. 135-145.

Kopardekar, P., Rhodes, J., Schwartz, A., Magyarits, S., and Willems, B. 2008. Relationship of maximum manageable air traffic control complexity and sector capacity. In 26th International Congress of the Aeronautical Sciences (ICAS 2008), and AIAA-ATIO-2008-8885, Anchorage, Alaska, Sept pp. 15-19.

Lee, J.D., and Moray, N. 1992. Trust, control strategies, and allocation of function in human machine systems. Ergonomics, 22: 671-691.

Lee, J.D., and Moray, N. 1994. Trust, self-confidence, and operators' adaptation to automation. Int. J. Hum. Comput. Stud. 40: 153-184. doi :10.1006/ijhc.1994.1007.

Moray, N., and Inagaki, T. 1999. Laboratory studies of trust between humans and machines in automated systems. Trans. Inst. Meas. Control 21(4-5): 203-211. doi: 10.1177/014233129902100408.

Muir, B.M. 1994. Trust in automation: Part I. Theoretical issues in the study of trust and human intervention in automated systems. Ergonomics, 37(11): 1905-1922. doi: 10.1080/00140139408964957.

Parasuraman, R., Molloy, R., and Singh, I.L. 1993. Performance consequences of automation-induced complacency. Inter. J. Aviat. Psychol. 3(1): 1-23. doi: 10.1207/s15327108ijap0301_1.

Parasuraman, R., and Riley, V. 1997. Humans and automation: Use, misuse, disuse, abuse. Hum. Factors, 39 : 230-253. doi: 10.1518/001872097778543886.

Pestana M.E. 2011. Flying Unmanned Aircraft: A Pilot's Perspective. Available from: https://ntrs.nasa.gov/archive/ nasa/casi.ntrs.nasa.gov/20110011979.pdf.

Radišić, T., Andraši, P., Novak, D., Juričić, B., and Antulov-Fantulin, B. 2020. Air Traffic Complexity as a Source of Risk in ATM. In Risk Assessment in Air Traffic Management. IntechOpen. Available from https:// www.intechopen.com/books/risk-assessment-in-air-traffic-management/air-traffic-complexity-as-a-source-of-riskin-atm.

Research and Innovative Technology Administration [RITA]. 2013. Unmanned aircraft system (UAS) service demand 2015-2035: Literature review \& projections of future usage. Department of Transportation Report No DOT-VNTSCDoD-13-01. Available from https://www.hsdl.org/?search\&exact=United+States.+Department+of+Transportation.+ Research+and+Innovative+Technology+Administration\&searchfield=publisher\&collection=limited\&submitted= Search\&advanced $=1 \&$ release $=0$.

Rice, S., Winter, S.R., Capps, J., Trombley, J., Robbins, J.R., and Milner, M.N. 2020. Creation of Two Valid Scales: Willingness to Fly in an Aircraft and Willingness to Pilot an Aircraft. Inter. J. Aviation Aeron. Aerospace, 7(1): 1-21. 
Richards, D., and Edgell, S. 2017. Attitudes toward UAV integration into the National Airspace System. In 17th AIAA Aviation Technology, Integration, and Operations Conference. p. 3939.

Vempati, L., Winter, S.R., Rice, S., Gawron, V., and Robbins, J. M 2021. Pilots' Willingness to Operate in Unmanned Aircraft System Integrated Airspace. Inter. J. Aerospace Psychol. 343-359. doi: 10.1080/24721840.2021.1896365.

\section{Appendix A}

\section{Trust, perceived risk, and willingness to pilot an aircraft scale}

Trust scale adapted from Jian et al. (2000).

I feel the autonomous system is
reliable
I feel the autonomous system
has integrity
I believe the autonomous system
is dependable
system
I have confidence in autonomous
system

Perceived Risk Scale adapted from Clothier et al. (2015).

I feel the autonomous system is
dangerous
I feel the autonomous system is
risky
I feel the autonomous system is
a liability
I feel the autonomous system is
I feel the autonomous system is
a hazard


Willingness to pilot an aircraft adapted from Rice et al. (2020).

\begin{tabular}{l} 
I would feel safe piloting an \\
aircraft in this situation \\
\hline $\begin{array}{l}\text { I would be willing to pilot an } \\
\text { aircraft in this situation }\end{array}$ \\
I would have no fear piloting an \\
aircraft in this situation \\
I would have no problem piloting \\
an aircraft in this situation \\
\hline I feel confident piloting an \\
aircraft in this situation \\
\hline I would be confident piloting an \\
aircraft in this situation \\
\hline I would be happy piloting an \\
aircraft in this situation
\end{tabular}

\section{Appendix B}

\section{Scenarios}

\section{Scenario 1}

Imagine a scenario where you are flying an aircraft equipped for visual flight rules (VFR) under visual meteorological conditions (VMC) in the airspace listed below. The weather conditions are visibility at least 10 statute miles or better and the sky conditions are clear. For your route of flight, you learn that urban air mobility (UAM) passenger transport operations carrying 2-4 passengers will be operating in your vicinity.

The UAM aircraft is fully autonomous with a pilot on board monitoring the operation.

The UAM operations are fully integrated with other manned aircraft operations.

\section{Scenario 2}

Imagine a scenario where you are flying an aircraft equipped for visual flight rules (VFR) under visual meteorological conditions (VMC) in the airspace listed below. The weather conditions are visibility at least 10 statute miles or better and the sky conditions are clear. For your route of flight, you learn that urban air mobility (UAM) passenger transport operations carrying 2-4 passengers will be operating in your vicinity.

The UAM aircraft is fully autonomous with a remote pilot monitoring the operation. The UAM operations are fully integrated with other manned aircraft operations. 